\title{
Anxiety/depression and orofacial myofacial disorders as factors associated with TMD in children
}

\section{Raquel Aparecida Pizolato(a) Frederico Silva de Freitas- Fernandes $^{(b)}$ \\ Maria Beatriz Duarte Gavião(c)}

\footnotetext{
(a) Departamento de Odontologia Social, Faculdade de Odontologia de Piracicaba, Univ Estadual de Campinas - UNICAMP, Piracicaba, SP, Brazil.
}

(b) Departamento de Odontologia I, Faculdade de Odontologia, Univ Federal do Maranhão - UFMA, São Luís, MA, Brazil.

(c) Departamento de Odontologia Infantil, Faculdade de Odontologia de Piracicaba, Univ Estadual de Campinas - UNICAMP, Piracicaba, SP, Brazil.
Declaration of Interests: The authors certify that they have no commercial or associative interest that represents a conflict of interest in connection with the manuscript.

\section{Corresponding Author:}

Maria Beatriz Duarte Gavião

E-mail:mbgaviao@fop.unicamp.br

Submitted: Oct 27, 2012

Accepted for publication: Dec 17, 2012

Last revision: Jan 04, 2013

\begin{abstract}
The purpose of this study was to evaluate factors associated with temporomandibular disorder (TMD) in children. TMD clinical signs were evaluated using the Research Diagnostic Criteria for TMD (RDC/TMD; axis I), and subjective symptoms were evaluated using a structured questionnaire. Eighty-two children were selected, 40 with TMD ( 19 boys and 21 girls, mean age $9.84 \pm 1.53$ and $9.71 \pm 1.30$ years, respectively) and 42 without TMD (21 boys and 21 girls, mean age $10.27 \pm 1.63$ and $9.9 \pm 1.37$ years, respectively). Intra- and extra-oral examinations were carried out to determine the myofunctional characteristics of the masticatory system. The Hospital Anxiety and Depression Scale (HADS) was used to assess anxiety and depression. Data were analyzed using descriptive statistics and bivariate analysis followed by multiple logistic regression $(\alpha=0.05)$. In bivariate analysis, the variables of open lips, anxiety, and depression had a $\mathrm{p}$ value $<0.15$ and were entered into the multivariate logistic regression model. The most significant predictor factors were the presence of open lips (odds ratio $=5.42$, $\mathrm{p}=0.034$ ) and anxiety (odds ratio $=18.59, \mathrm{p}<0.001$ ). Thus, anxiety levels and open lips were associated with TMD in children. Owing to the cross-sectional design of the present study, the associations observed may have a bidirectional relationship.
\end{abstract}

Descriptors: Temporomandibular Joint Disorders; Anxiety; Depression; Child; Risk Factors.

\section{Introduction}

Temporomandibular disorder (TMD) is a generic term for a number of clinical signs and symptoms, involving pain in the preauricular area or the muscles of mastication, limitation or deviations in the mandibular range of motion, noise and pain in the temporomandibular joint (TMJ) during mandibular function, and headaches. ${ }^{1}$ Several studies have shown that functional disruption of the masticatory system in children and adolescents is common, and its occurrence increases with age. ${ }^{1,2}$ TMD has multifactorial etiology; oral parafunctions (such as bruxism), trauma to the mandible or $\mathrm{TMJ},{ }^{3}$ and psychosocial factors ${ }^{4}$ are known etiological or contributing factors. Furthermore, anxiety and depression have been observed in pediatric populations with signs and symptoms of TMD..$^{5,6}$

TMD has also been associated with orofacial myofunctional disorders (OMD). ${ }^{5,-9}$ OMD may contribute to TMD by causing disequilibri- 
um of TMJ function, which may lead to compensatory muscle behaviors that consequently aggravate the problem. ${ }^{8,9}$ Thus, clinical examination directly focusing on orofacial myofunctional conditions is important in TMD studies. ${ }^{9}$

Functional disorders of the stomatognathic system may occur in children ${ }^{1,10}$ but are infrequently diagnosed and may consequently result in TMD. In this way, awareness of the early signs and symptoms of TMD—focusing on the assessment of masticatory muscles, the TMJ, and associated structures-may reveal if symptoms are TMD in origin. ${ }^{11}$ Moreover, because TMD may have psychological underpinnings, accurate diagnosis is critical. Thus, the aim of the present study was to investigate if OMD and psychological characteristics, such as anxiety and depression, are associated with TMD in children.

\section{Methodology}

Children aged 8 to 12 years were recruited from two public schools in Piracicaba, SP, Brazil. The Ethics Committee of the Piracicaba Dental School approved this research (Protocol no. 034/2006). Parents or guardians of the children were informed of the purpose of the research, and written informed consent was obtained.

Initially, 350 children atending those schools were invited to participate. A total of 152 children ( 78 boys and 74 girls, mean age $10.05 \pm 1.39$ years) were enrolled in the study after examination and application of the following inclusion and exclusion criteria.

1. inclusion criteria:

- healthy state or the absence of systemic problems with the masticatory system,

- presence of all primary and/or permanent teeth without anomalies or alterations in form, structure, or number;

2. exclusion criteria:

- neurological disturbances (such as cerebral palsy, movement disorders, speech and language disorders, and behavioral/cognitive syndromes),

- menarche,

- any type of orthodontic treatment prior to or during the research examination period, and

- uncooperative behaviors.
The children were assessed for the presence of TMD using axis I of the Research Diagnostic Criteria for TMD (RDC/TMD) by a single calibrated examiner. The intra-examiner reliability was calculated by twice examining 20 children not participating in the study with an interval of 7 days, and a Kappa value of 0.91 was obtained. TMD was verified by pain upon palpation, mandibular range of motion $(\mathrm{mm})$, pain during mandibular movements, sounds from the TMJ, and tenderness induced by muscle and joint palpation. A self-reported questionnaire was used to assess subjective symptoms, including pain in the jaws during function, frequent headaches (more than once a week), stiffness/fatigue in the jaws, difficulty in opening the mouth wide, grinding teeth, and TMJ sounds. Each question required a "yes" or "no" answer. Three questions about subjective TMD symptoms included in the axis II of the RDC/TMD criteria $^{12}$ were considered for TMD diagnosis: ${ }^{13,14}$

- (3) Have you had pain in the face, jaw, temple, in front of the ear, or in the ear in the past month? (Obs.: Only pains of unknown etiology were considered)

- (14.a) Have you ever had your jaw lock or catch so that it would not open all the way?

- (14.b) Was this limitation in jaw opening severe enough to interfere with your ability to eat?

The RDC/TMD criteria classify the forms of TMD into three diagnostic categories:

- Group I: muscle disorders (Group Ia with myofascial pain; Group Ib with myofascial pain with limited opening);

- Group II: disc displacement (Group IIa with reduction; Group IIb without reduction with limited opening; Group IIc without reduction but without limited opening);

- Group III: arthralgia (Group IIIa) or arthritis (Group IIIb/IIIc).

After the assessments above, 82 children were selected considering the presence or absence of TMD:

- 40 children with TMD (19 boys, 21 girls; mean age $9.84 \pm 1.53$ and $9.71 \pm 1.30$ years, respectively) and 
- 42 without TMD (21 boys, 21 girls, mean age $10.27 \pm 1.63$ and $9.9 \pm 1.37$ years, respectively).

The other 70 children were excluded because they were not diagnosed with TMD according to the RDC/TMD.

\section{Hospital Anxiety and Depression Scale (HADS)}

The level of anxiety and depression was rated using the HADS, which consists of seven items for depression (HADSd) and seven items for anxiety (HADSa). HADS was first described by Zigmond and Snaith, ${ }^{15}$ and a Portuguese version was validated by Botega et al. ${ }^{16}$ The main characteristic of HADS is that items covering somatic symptoms of anxiety and depression have been eliminated. By defining cutoff values, the HADS subscale can be used to rate mental disorders:

- 0 to 7 = normal;

- 8 to $10=$ mild;

- 11 to $14=$ moderate; and

- 15 to $21=$ severe $^{16}$.

HADS has been extensively applied and has well-established psychometric properties. ${ }^{17}$

\section{Evaluation of orofacial myofunctional characteristics}

The physical aspects of the lips, tongue, and mentalis muscle at rest were observed, as well as the characteristics of breathing, chewing, swallowing, and tongue movements, using visual inspection and palpation by a speech pathologist (R.A.P). ${ }^{13}$

Orofacial myofunctional characteristics were considered as follows.

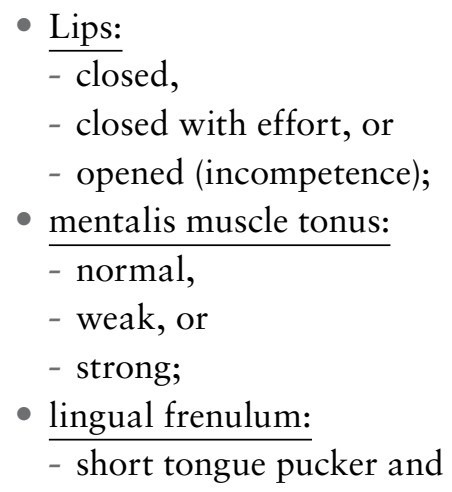

- restricted movement (exclusion criterion);

- tongue function:

- adequate resistance (determined using a spatu-

la) or

- altered (decreased resistance);

- tongue posture at rest:

- normal (sitting in the region of the incisive papilla) or

- altered position (sitting on the floor of the mouth, between the teeth, or tongue pressing forward);

- breathing: expiratory nasal permeability (tested using a Glatzel metal plate); permeability was described as

- oral,

- nasal, or

- mixed breathing, considering right/left nasal air flow;

- chewing: considered

- adequate when bilaterally alternate chewing was observed and

- inadequate when preferential (only one side) chewing was noted $(\mathrm{a} 2 \times 2-\mathrm{cm}$ piece of bread was used), without compensatory head movements and lip closure;

- swallowing: the child was instructed to swallow two pieces of bread $(2 \times 2 \mathrm{~cm})$ in his/her habitual manner followed by two portions of water while the examiner palpated the hyoid bone and the larynx region; bread and water swallowing were evaluated separately; the deglutition pattern was considered abnormal when one or more of the following characteristics were noted: ${ }^{7}(1)$ first evaluation: orbicularis oris and/or mentalis muscle contractions, forward head movement, and (2) second evaluation: the child's lips were slightly opened; the tongue position at the exact moment of swallowing was considered as follows:

- tongue pressing anterior incisors,

- lateral and/or anterior tongue thrust (tongue between the teeth), and

- lower lip pressing maxillary incisors.

\section{Statistical analysis}

The prevalence of TMD was calculated for the entire sample according to RDC/TMD. The depen- 
Table 1 - Bivariate analysis regarding associations among

TMD, malocclusion, gender, dentition, orofacial myofunctional disorders, anxiety, and depression.

\begin{tabular}{|c|c|c|c|c|}
\hline \multirow{2}{*}{\multicolumn{2}{|c|}{ Variables }} & \multicolumn{2}{|c|}{ TMD } & \multirow[b]{2}{*}{$p$-value } \\
\hline & & \multirow{2}{*}{$\begin{array}{c}\text { Yes } \\
n=40 \\
21(52.5)\end{array}$} & \multirow{2}{*}{$\begin{array}{c}\text { No } \\
n=42 \\
21(50)\end{array}$} & \\
\hline \multirow{2}{*}{ Gender } & Girls & & & \multirow{2}{*}{0.996} \\
\hline & Boys & 19 (47.5) & $21(50)$ & \\
\hline \multirow{2}{*}{ Open lips } & Present & $19(47.5)$ & $8(19.04)$ & \multirow{2}{*}{0.012} \\
\hline & Absent & $21(52.5)$ & $34(80.09)$ & \\
\hline \multirow{2}{*}{ Lip interposition } & No & $19(47.5)$ & $25(59.52)$ & \multirow{2}{*}{0.384} \\
\hline & Yes & $21(52.5)$ & $17(40.47)$ & \\
\hline \multirow{2}{*}{$\begin{array}{l}\text { Tongue in the floor of the } \\
\text { mouth }\end{array}$} & No & 15 (37.5) & $20(47.61)$ & \multirow{2}{*}{0.482} \\
\hline & Yes & $25(62.5)$ & $22(52.38)$ & \\
\hline \multirow{2}{*}{ Tongue hypofunction } & No & $16(40)$ & $20(47.61)$ & \multirow{2}{*}{0.637} \\
\hline & Yes & $24(60)$ & $22(52.38)$ & \\
\hline \multirow{2}{*}{$\begin{array}{c}\text { Tongue between dental } \\
\text { arches }\end{array}$} & No & $15(37.5)$ & $19(45.23)$ & \multirow{2}{*}{0.626} \\
\hline & Yes & $25(62.5)$ & $23(54.76)$ & \\
\hline \multirow{2}{*}{ Anterior tongue pressure } & No & $36(90)$ & 35 (83.33) & \multirow{2}{*}{0.575} \\
\hline & Yes & $4(10)$ & $7(16.66)$ & \\
\hline \multirow{2}{*}{$\begin{array}{c}\text { Rigid mentalis muscle } \\
\text { tonus }\end{array}$} & No & $11(27.5)$ & $11(26.19)$ & \multirow{2}{*}{0.908} \\
\hline & Yes & 29 (72.5) & $31(73.80)$ & \\
\hline \multirow{2}{*}{$\begin{array}{c}\text { Orbicularis oris muscle } \\
\text { contraction }\end{array}$} & No & $11(27.5)$ & $11(26.19)$ & \multirow{2}{*}{0.908} \\
\hline & Yes & $29(72.5)$ & $31(73.80)$ & \\
\hline \multirow{2}{*}{ Anxiety } & No & $17(42.5)$ & $39(92.85)$ & \multirow{2}{*}{$<0.0001$} \\
\hline & Yes & $23(57.5)$ & $3 \quad(7.14)$ & \\
\hline \multirow{2}{*}{ Depression } & No & $31(77.5)$ & $39(92.85)$ & \multirow{2}{*}{0.098} \\
\hline & Yes & $9(22.5)$ & $3(7.14)$ & \\
\hline
\end{tabular}

dent variable (TMD diagnosis) was dichotomized as "yes" or "no". Bivariate analysis was carried out to verify the influence of the independent variables (gender, habits, anxiety, depression, and orofacial myofunctional characteristics) on the dependent variable concerning TMD, using the chi-square test at the $5 \%$ significance level.

Next, multiple logistic regression analysis using the stepwise procedure was conducted to identify the factors associated with TMD. Only independent variables that showed a significant association (determined through the bivariate analysis, $\mathrm{p}<0.15$ ) were selected for the regression analysis. The logistic regression models were adjusted according to the odds ratio, 95\% confidence intervals, and significance levels. All statistical tests were carried out using SPSS 13.0 software (Statistical Package for Social Sciences, IBM, Chicago, USA) at the 5\% significance level.

\section{Results}

The prevalence of TMD among girls and boys was similar $(52.5 \%$ and $47.5 \%$, respectively). Table 1 shows the distribution of children according to the independent variables that were significantly associated with TMD. Bivariate analysis showed that TMD was significantly associated with anxiety and open lips. For depression, the p-value was below 0.15 and was considered an independent variable in the multiple logistic regression model. Open lips and anxiety remained as associated variables in the respective model (Table 2). Children with open lips 
Table 2 - Multivariate stepwise forward proceeding analysis for TMD presence and independent variables.

\begin{tabular}{c|r|r|c|c}
\hline Variables & $\begin{array}{c}\text { TMD } \\
\mathrm{n}(\%)\end{array}$ & $\begin{array}{c}\text { Odds } \\
\text { ratio }\end{array}$ & $\begin{array}{c}\text { Confidence } \\
\text { interval }\end{array}$ & -level \\
\hline Open lips & $21(52.5)$ & 6.10 & $1.83-20.40$ & 0.003 \\
\hline Anxiety & $23(57.5)$ & 18.06 & $4.29-76.03$ & $<.0001$ \\
\hline Depression & $9(22.5)$ & 2.08 & $0.35-12.25$ & 0.418 \\
\hline
\end{tabular}

were six times more likely to develop TMD than those with adequate lip closure, whereas those with high levels of anxiety were 18 times more likely to develop TMD.

\section{Discussion}

TMD is increasingly common among children worldwide, and thus early diagnosis is critical to minimizing its long-term effects. TMD possibly could be prevented through a better understanding of its etiological factors. ${ }^{13}$ Therefore, our present cross-sectional study investigated the impact of several etiological factors on the signs and symptoms of TMD in children.

No association was found between gender and TMD that could be attributed to the childrens' age, i.e., they were pre-pubertal and thus not influenced by reproductive hormones, which are associated with an increased risk of TMD pain. ${ }^{18}$ In this context, Pereira et al. ${ }^{19}$ found gender-related disparities at the beginning of puberty with a higher prevalence of TMD in girls despite the observation that menarche has no effect on TMD diagnosis. Nilsson ${ }^{20}$ observed that TMD pain seems to have a greater impact on girls than boys. In addition, the most prominent gender differences in pain are seen in 20- to 40 -year-old women. ${ }^{21}$

The present findings show that anxiety is significantly associated with TMD, in agreement with other studies. ${ }^{5,6,9,13}$ Individuals with TMD are more emotionally reactive to environmental challenges than those without TMD ${ }^{6}$ and approximately onethird of patients seeking treatment for TMD present with signs and symptoms of depression. ${ }^{20,22}$ The results of the present study, however, revealed no association between depression and TMD, which differs from the findings of previous studies that re- ported such an association in adolescents. ${ }^{5,7,19}$ Children, in general, can be assumed to develop anxiety more than depression, which usually appears more frequently in adolescence. It is important to consider that adolescents experience many developmental challenges as they strive to break away from their parents, become independent, and establish their own identities. ${ }^{1}$ However, childhood involves a setting filled with the burden of learning activities, which may contribute to a child's high level of anxiety. In the present study, considering TMD as the dependent variable, children with anxiety were about 18 times more likely to develop TMD than those without anxiety, confirming the role of psychological factors in TMD reported previously. ${ }^{6,10}$ Nonetheless, the causality of the observed associations must be carefully interpreted because the cross-sectional study design does not allow us to establish whether the characteristic developed before or after TMD onset, thus precluding assumptions about possible causal effects. ${ }^{3}$ Despite the different ages and study designs, our findings are similar to the longitudinal study of Aggarwal et al., ${ }^{23}$ who reported that four psychosocial measures can distinguish cases with chronic orofacial pain from controls in univariate analyses; however, only anxiety remained a significant predictor in the multivariate approach.

Open lips were associated with TMD, confirming that OMD may compromise the balance of the stomatognathic system, ${ }^{9}$ corroborating previous studies reporting an association between OMD and TMD. ${ }^{5,7}$ The absence of lip sealing may lead to TMD owing to inadequate positioning of the mandible and an effort to maintain the balance of the stomatognathic system, ${ }^{9}$ which depends on the structural and physiological tolerance of each individual. In this context, if labial dysfunction coexists with TMD symptoms, hypofunctioning or hyperfunctioning lips could result. In the first case, other muscles would need to participate to permit anterior sealing of the oral cavity. In the second case, excessive muscle activity may play a role. ${ }^{24}$ On the other hand, the duration of TMD may influence OMD, because TMD signs and symptoms may also aggravate OMD over time owing to the need for adaptations or compensations involving the muscles and 
the stomatognathic functions. ${ }^{9}$ Thus, it was not possible to establish whether the open lips were established before or after TMD onset, and we cannot draw conclusions about possible causal effects as considered by Orbach et al. ${ }^{3}$ in relation to clinical findings as risk factors for chronic TMD. Although the other orofacial myofunctional characteristics showed no association with TMD diagnosis, they must be considered during evaluation of the stomatognathic system, mainly in children, because significant differences have been observed between adults with TMD and healthy adults in the scores of mandibular posture, facial symmetry, tongue and mandible mobility, and the functions of deglutition and mastication. ${ }^{24}$

Furthermore, other factors such as masticatory system development and parafunctional oral habits have been reported as indicators of TMD in children. ${ }^{5}$ This topic is controversial in the literature because no particular morphological or functional occlusal factor is significantly associated with TMD. ${ }^{19}$ A new scenario for the risk of developing TMD arises when considering gene-environment interactions. ${ }^{25,26}$

Shortcomings in the current study must be con-

\section{References}

1. Bonjardim LR, Gavião MB, Pereira LJ, Castelo PM. Signs and symptoms of temporomandibular disorders in adolescents. Braz Oral Res. 2005 Apr-Jun;19(2):93-8.

2. Castelo PM, Gavião MBD, Pereira LJ, Bonjardim LR. Relationship between oral parafunctional/nutritive sucking habits and temporomandibular joint dysfunction in primary dentition. Int J Paediatr Dent. 2005 Jan;15(1):29-36.

3. Ohrbach R, Fillingim RB, Mulkey F, Gonzalez Y, Gordon S, Gremillion $\mathrm{H}$, et al. Clinical findings and pain symptoms as potential risk factors for chronic TMD: descriptive data and empirically identified domains from the OPPERA case-control study. J Pain. 2011 Nov;12(11 Suppl):T27-45.

4. Fillingim RB, Ohrbach R, Greenspan JD, Knott C, Dubner $\mathrm{R}$, Bair E, et al. Potential psychosocial risk factors for chronic TMD: descriptive data and empirically identified domains from the OPPERA case-control study. J Pain. 2011 Nov;12(11 Suppl):T46-60.

5. Restrepo CG, Vásquez LM, Alvarez M, Valencia I. Personality traits and temporomandibular disorders in a group of children with bruxing behavior. J Oral Rehabil. 2008 Aug;35(8):58593. sidered. First, the study was based on a convenient sample of limited size. Consequently, extrapolation of the findings to the general population requires caution. Second, this was a cross-sectional study, and the fluctuation of TMD signs and symptoms requires longitudinal studies to observe the onset. Furthermore, the cross-sectional characteristics cannot determine the causality of the observed associations. However, these shortcomings do not detract from the importance of recognizing children with a predisposition to disorders of the stomatognathic system. Thus, interactions among all possible etiologic factors must be addressed.

\section{Conclusion}

In the present study, anxiety levels and open lips appeared to be associated with TMD in children. Owing to the cross-sectional design of the present study, the observed associations may have a bidirectional relationship.

\section{Acknowledgements}

Scholarships for the first and second authors from CAPES (Brazil) are gratefully acknowledged.

6. Bonjardim LR, Gavião MB, Pereira LJ, Castelo PM. Anxiety and depression in adolescents and their relationship with signs and symptoms of temporomandibular disorders. Int J Prosthodont. 2005 Jul-Aug;18(4):347-52.

7. Genaro KF, Berretin-Felix G, Rehder MIBC, Marchesan IQ. Avaliação miofuncional orofacial: protocolo MBGR. Rev Cefac. 2009 Abr-Jun;11(2):237-55.

8. Berretin-Felix G, Genaro KF, Trindade IE, Trindade Júnior AS. Masticatory function in temporomandibular dysfunction patients: electromyographic evaluation. J Appl Oral Sci. 2005 Dec;13(4):360-5.

9. Ferreira CLP, Silva MAMR, Felício CM. Orofacial myofunctional disorder in subjects with temporomandibular disorder. Cranio. 2009 Oct;27(4):268-74.

10. Tecco S, Festa F. Prevalence of signs and symptoms of temporomandibular disorders in children and adolescents with and without crossbite. World J Orthod. 2010 Spring;11(1):37-42.

11. Howard JA. Temporomandibular joint disorders in children. Dent Clin North Am. 2013 Jan;57(1):99-127.

12. Dworkin SF, LeResche L. Research diagnostic criteria for temporomandibular disorders:review, criteria, examinations 
and specifications, critique. J Craniomandib Disord. 1992 Fall;6(4):301-55.

13. Pizolato RA, Fernandes FSF, Gavião MBD. Deglutition and temporomandibular disorders in children. Minerva Stomatol. 2009 Nov-Dec;58(11-12):567-76.

14. Pizolato RA, Fernandes FS, Gavião MB. Speech evaluation in children with temporomandibular disorders. J Appl Oral Sci. 2011 Oct;19(5):493-9.

15. Zigmond AS, Snaith RP. The hospital anxiety and depression scale. Acta Psychiatr Scand. 1983 Jun;67(6):361-70.

16. Botega NJ. Bio MR, Zomingnani MA, Garcia Jr C, Pereira WAB. Mood disorders among inpatients in ambulatory and validation of the anxiety and depression scale HAD. Rev Saude Publica. 1995 Oct;29(5):355-63. Portuguese.

17. Mykletun A, Stordal E, Dahl AA. The hospital anxiety and depression scale (HADS): Factor structure, item analyses, and internal consistency in a large population. Br J Psychiatry. 2001 Dec;179(1):540-4.

18. LeResche L, Mancl LA. Drangsholt MT. Relationship of pain and symptoms to pubertal development in adolescent. Pain. 2005 Nov;118(1-2):201-9.

19. Pereira LJ, Cenci-Pereira T, Del Bel Cury AA, Pereira SM, Pereira AC, Ambrosano GMB, et al. Risk Indicators of Temporomandibular Disorder Incidence in early adolescence. Pediatr Dent. 2010 Jul-Ag;32(4):324-8.
20. Nilsson IM. Reliability, validity, incidence and impact of temporomandibular pain disorders in adolescents. Sweed Dent J Suppl. 2007;(183):7-86.

21. Meisler JG. Chronic pain conditions in women. J Womens Health. 1999 Apr;8(3):313-20.

22. Manfredini D, Landi N, Bandettini Di Poggio A, Dell'Osso L, Bosco M. A critical review on the importance of psychological factors in temporomandibular disorders. Minerva Stomatol. 2003 Jun;52(6):321-30.

23. Aggarwal VR, Macfarlane GJ, Farragher TM, McBeth J. Risk factors for onset of chronic oro-facial pain-results of the North Cheshire oro-facial pain prospective population study. Pain. 2010 May;149(2):354-9.

24. Felício CM, Oliveira MM, Silva MA. Effects of orofacial myofunctional therapy on temporomandibular disorders. Cranio. 2010 Oct;28(4):249-59.

25. Michelotti A, Iodice G. The role of orthodontics in temporomandibular disorders. J Oral Rehabil. 2010 May;37(6):411-29.

26. Smith SB, Maixner DW, Greenspan JD, Dubner R, Fillingim $\mathrm{RB}, \mathrm{Oh}$ rbach R, et al. Potential genetic risk factors for chronic TMD: genetic associations from the OPPERA case control study. J Pain. 2011 Nov;12(11 Suppl):T92-101. 